\title{
Novel nanoscale transfer printing technique for precise positioning of nanowire lasers
}

Antonio Hurtado, Dimitars Jevtics, Benoit Guilhabert, Qian Gao, Hark H. Tan, Chennupati Jagadish, and Martin D. Dawson

Semiconductor nanowires, with lasing emission at room temperature, can be transferred in a controlled way to specific locations on diverse substrates and organized into bespoke spatial patterns.

Semiconductor nanowire (NW) lasers provide coherent light sources with highly localized emission and extremely small footprints. Such lasers may thus have the potential to revolutionize the field of photonics. ${ }^{1-3}$ Indeed, NW lasers are expected to play a key role in future optoelectronic systems, i.e., with applications in nanophotonic integrated circuits for on-chip communications and computing, in ultrasmall laser sensors for healthcare, and in light-cell interfaces for biological sciences. ${ }^{1-3}$ The extremely small dimensions of NW lasers, however, mean that it is technologically challenging to accurately manipulate and integrate them into functional systems. Their transition from laboratory environments to real-life, industrially relevant products is thus limited.

In the past, several approaches have been proposed for the manipulation of NWs. These include optical tweezers, ${ }^{4}$ Langmuir-Blodgett assembly processes, ${ }^{5}$ the use of microscope probes, ${ }^{6}$ or contact printing techniques. ${ }^{7}$ All these techniques, however, have associated problems. For example, the NWs must be in solution, complex equipment is required, they provide reduced positioning accuracy, they do not allow individual NWs to be manipulated, or heterogeneous NWs cannot be integrated within the same system. The precise, simple, and efficient manipulation of single-NW lasers is thus still to be realized.

At the Institute of Photonics (IOP) of the University of Strathclyde, UK, we have thus developed a new technique-known as nanoscale transfer printing (nano-TP) - to tackle the challenge of single-NW laser manipulation. ${ }^{8,9}$ Transfer printing technology (originally introduced by John Rogers at the University of

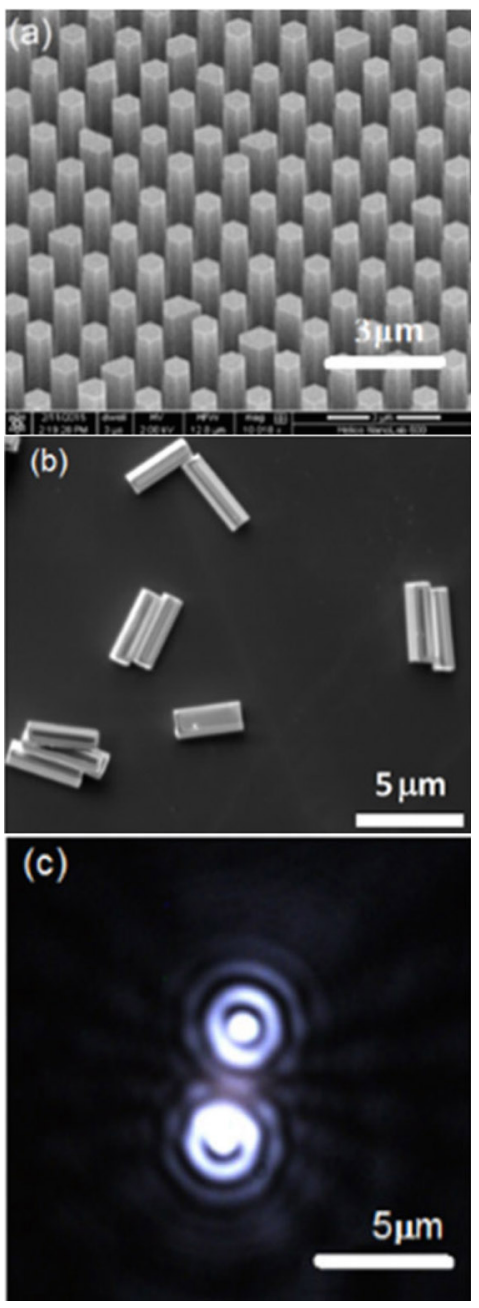

Figure 1. Scanning electron images of indium phosphide (InP) nanowire (NW) lasers that are (a) vertically aligned (as-grown) on an InP substrate and (b) randomly scattered on a silicon (Si) surface. (c) Image of lasing emission (in the 840-890nm range) from the NW lasers. $^{8}$

Continued on next page 
Illinois ${ }^{10}$ ) involves the use of polymer stamps to capture semiconductor structures in a controlled manner and to subsequently release them onto diverse substrates. This approach provided a revolutionary platform for hybrid fabrication of a wide range of novel optoelectronic systems (e.g., semiconductor lasers printed on silicon substrates ${ }^{11}$ and LEDs printed on flexible and diamond substrates ${ }^{12}$ ). In turn, these systems had a large impact in many different technologies, such as visible light communications, flexible optoelectronics, and photonic integrated circuits.

In our nano-TP technique we use bespoke polymer microstamps ( $\mu$-stamps), which have reduced dimensions and controlled shapes, to capture/release indium phosphide (InP) NW lasers. ${ }^{13}$ To fabricate (at the Australian National University) the NW lasers we used in our work, we grew vertically aligned InP NWs on an InP substrate, as shown in Figure 1(a). Before performing our nano-TP study, we removed the NWs from the growth substrate and used mechanical means to randomly scatter them onto a silicon (Si) substrate: see Figure 1(b). The final lasers have a lasing emission-see Figure 1(c) — at room temperature in the $\sim 840-890 \mathrm{~nm}$ wavelength range. ${ }^{13}$

(a)
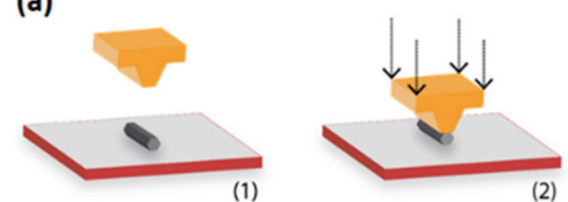

(2)

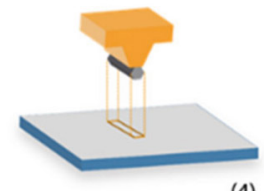

(4)

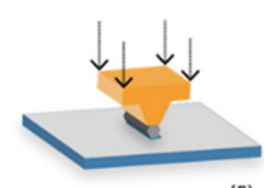

(5)

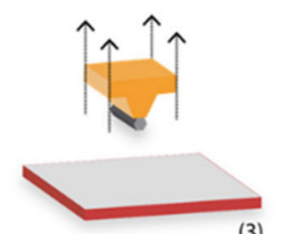

(3)

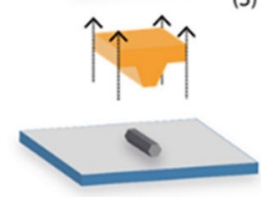

(6)
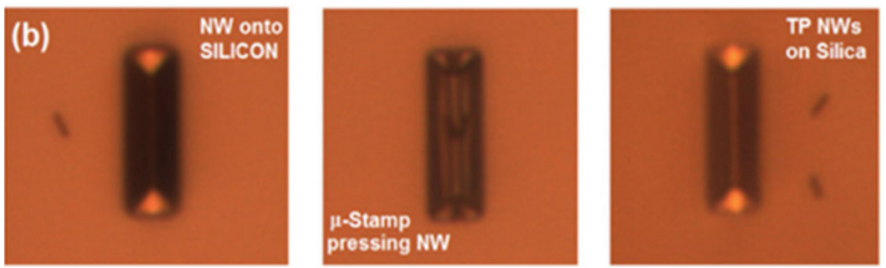

Figure 2. (a) Schematic diagrams illustrating the six stages of the nanoscale transfer printing (nano-TP) technique, i.e., showing (1) alignment of the NW and microstamp ( $\mu$-stamp), (2) surface (growth substrate) contact, (3) NW lift-off, (4) alignment of the $\mu$-stamp with the receiving substrate, (5) surface (receiving substrate) contact, and (6) NW release. (b) Sequence of microscope images illustrating the transfer of an InP NW laser $(5 \mu \mathrm{m}$ in length, 660nm in diameter) from a Si substrate (left) to a silica surface (right), through pressing with a v-shaped $\mu$-stamp. ${ }^{8}$
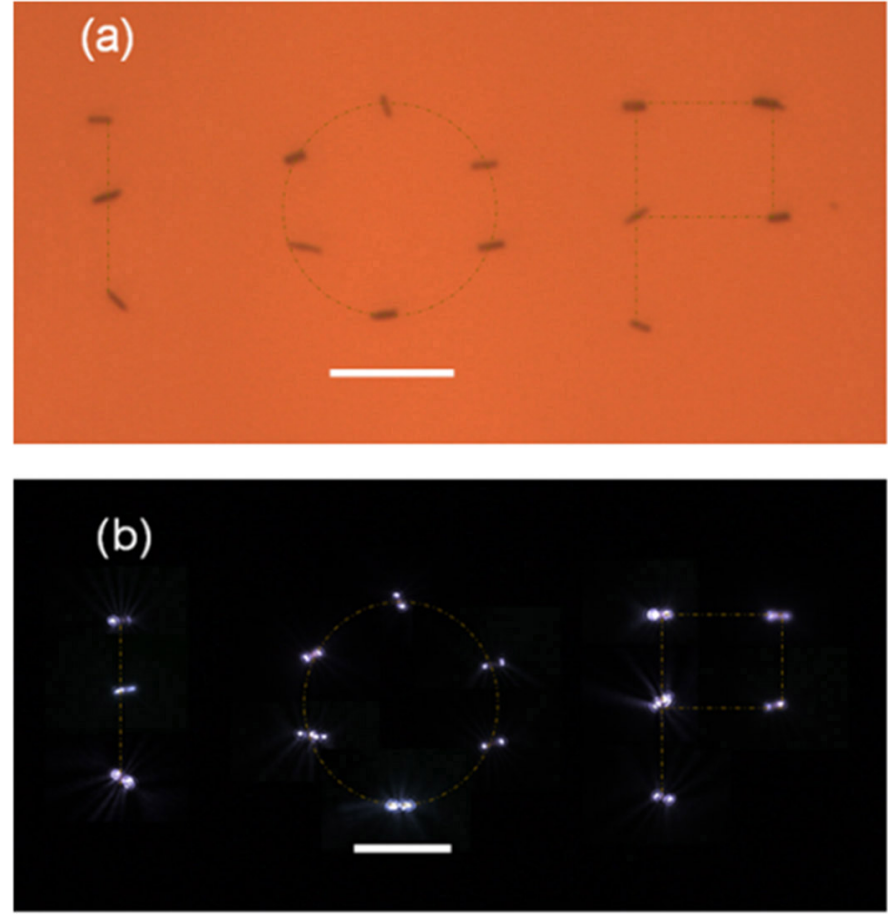

Figure 3. (a) Bright and (b) dark micrographs of an 'IOP' pattern formed from InP NWs on polydimethylsiloxane, through the use of nano-TP. Scale bar marks $20 \mu m .^{8}$

To fabricate our $\mu$-stamps, we used polydimethylsiloxane (PDMS), i.e., an elastomeric and adhesive polymer that conforms to the shape of the NW when pressed against it. The NW therefore adheres to the $\mu$-stamp and enables its capture. After lifting off the NW in our approach, we align the $\mu$-stamp (with the NW attached) at a targeted location on a secondary substrate (where the NW is then released). The different mechanisms and stages of our nano-TP process are illustrated in Figure 2. ${ }^{8}$

We have also used our nano-TP approach to demonstrate the precise transfer of InP NWs that have different dimensions (i.e., diameters of 435,660 , and $920 \mathrm{~nm}$ ) from a primary Si substrate to targeted locations on heterogeneous surfaces (e.g., PDMS, silica, or gold). ${ }^{8}$ We find that the NWs retain their lasing emission characteristics after the nano-TP process is completed. Furthermore, our technique permits the formation of micrometric spatial patterns from NW lasers on diverse substrates. For example, we show bright and dark micrographs of an 'IOP' pattern in Figure 3. We fabricated this pattern-in which all elements (i.e., NWs) kept their lasing emission-with the use of 435nmdiameter InP NWs. ${ }^{8}$ 
In summary, we have developed a novel transfer printing technique-known as nano-TP_that enables precise positioning of NW lasers onto heterogeneous substrates. We can also use our approach to organize the NW lasers into bespoke micrometric patterns. Notably, the NWs retain their lasing emission even after the TP protocols have been completed. Our enabling technology thus opens up potential new routes for accurate integration of NW lasers onto functional nanophotonic systems. Our future plans involve the use of nano-TP to incorporate NW lasers into waveguiding technological platforms, and to thus develop reduced footprint nanophotonic integrated circuits for applications in information and communication technologies (e.g., integrated nanoscale light sources in on-chip communications/computing systems) and healthcare (e.g., nanolaser integrated sensing modules).

Financial support for this work was provided by the University of Strathclyde (through the Strathclyde's Chancellor Fellowship Program), the Australian Research Council, and the UK's Engineering and Physical Sciences Research Council (grant EP/I029141/1). We thank the Australian National Fabrication Facility, ACT Node, for access to the growth facilities used in this work.

\section{Author Information}

Antonio Hurtado, Dimitars Jevtics, Benoit Guilhabert, and Martin D. Dawson

University of Strathclyde

Glasgow, United Kingdom

Antonio Hurtado has more than 10 years of research experience in photonics in the UK, US, and Spain. He was awarded two prestigious Marie Curie Fellowships by the European Commission and a Strathclyde Chancellor's Fellowship, after which he was appointed as lecturer in the University of Strathclyde's Institute of Photonics.

\section{Qian Gao, Hark H. Tan, and Chennupati Jagadish}

Australian National University

Canberra, Australia

\section{References}

1. R. Yan, D. Gargas, and P. Yang, Nanowire photonics, Nat. Photon. 3, pp. 569-576, 2009.

2. P. Yang, R. Yan, and M. Fardy, Semiconductor nanowire: what's next?, Nano Lett. 10, pp. 1529-1536, 2010.

3. S. W. Eaton, A. Fu, A. B. Wong, C.-Z. Ning, and P. Yang, Semiconductor nanowire lasers, Nat. Rev. Mater. 1, p. 16028, 2016.

4. P. J. Pauzauskie, A. Radenovic, E. Trepagnier, H. Shroff, P. Yang, and J. Liphardt, Optical trapping and integration of semiconductor nanowire assemblies in water, Nat. Mater. 5, pp. 97-101, 2006

5. S. Jin, D. Whang, M. C. McAlpine, R. S. Friedman, Y. Wu, and C. M. Lieber, Scalable interconnection and integration of nanowire devices without registration, Nano Lett. 4, pp. 915-919, 2004.

6. H. Xu, A. Hurtado, J. B. Wright, C. Li, S. Liu, J. J. Figiel, T.-S. Luk, et al., Polarization control in GaN nanowire lasers, Opt. Express 22, pp. 19198-19203, 2014.

7. Z. Fan, J. C. Ho, Z. A. Jacobson, R. Yerushalmi, R. L. Alley, H. Razavi, and A. Javey, Wafer-scale assembly of highly ordered semiconductor nanowire arrays by contact printing, Nano Lett. 8, pp. 20-25, 2008.

8. B. Guilhabert, A. Hurtado, D. Jevtics, Q. Gao, H. H. Tan, C. Jagadish, and M. D. Dawson, Transfer printing of semiconductor nanowires with lasing emission for controllable nanophotonic device fabrication, ACS Nano 10, pp. 3951-3958, 2016.

9. A. Hurtado, B. J. E. Guilhabert, M. J. Strain, N. Laurand, C. Jagadish, and M. D. Dawson, Nanoscale transfer printing for heterogeneous device integration. Presented at SPIE Photonics West 2017.

10. M. A. Meitl, Z.-T. Zhu, V. Kumar, K. J. Lee, X. Feng, Y. Y. Huang, I. Adesida, R. G. Nuzzo, and J. A. Rogers, Transfer printing by kinetic control of adhesion to an elastomeric stamp, Nat. Mater. 5, pp. 33-38, 2006.

11. X. Sheng, C. Robert, S. Wang, G. Pakeltis, B. Corbett, and J. A. Rogers, Transfer printing of fully formed thin-film microscale GaAs lasers on silicon with a thermally conductive interface material, Laser Photon. Rev. 9, pp. L17-L22, 2015.

12. A. J. Trindade, B. Guilhabert, E. Y. Xie, R. Ferreira, J. J. D. McKendry, D. Zhu, $\mathrm{N}$. Laurand, et al., Heterogeneous integration of gallium nitride light-emitting diodes on diamond and silica by transfer printing, Opt. Express 23, pp. 9329-9338, 2015.

13. Q. Gao, D. Saxena, F. Wang, L. Fu, S. Mokkapati, Y. Guo, L. Li, et al., Selective-area epitaxy of pure wurtzite InP nanowires: high quantum efficiency and room-temperature lasing, Nano Lett. 14, pp. 5206-5211, 2014. 